\title{
IPManager: A Microcomputer-Based DSS for Intellectual Property Management
}

\author{
Chuda Basnet \\ Les Foulds \\ Department of Management Systems \\ University of Waikato \\ New Zealand \\ Warren Parker \\ AgResearch \\ Ruakura Research Centre \\ Hamilton \\ Chuda@waikato.ac.nz \\ Lfoulds@waikato.ac.nz \\ Warren.Parker@agresearch.co.nz
}

\begin{abstract}
We describe a decision support system (DSS) that was developed for the management of the costs associated with the development of an organization's intellectual property (IP). IPManager is designed to aid managers of IP in creating or improving IP registration and maintenance strategies, and enables them to use their experience and preferences. The system can be used to analyze a wide variety of "what-if?" scenarios with potential cost impacts. The system is written in Microsoft Excel, within the Microsoft Windows environment. A case study is reported, discussing the environment that motivated its development, namely for the management of IP at AgResearch, Hamilton, New Zealand. The system provides a useful addition to the IP manager's toolkit and an interesting application of the DSS approach to a critical area of the knowledge economy.
\end{abstract}

\section{Introduction}

As the largest Crown Research Institute in New Zealand, AgResearch provides knowledge and best practice technology to pastoral agricultural industries. A strong focus on biotechnology and ecotechnology seeks to provide integrated life science solutions that create value for stakeholders and customers, wealth for New Zealand, and better health for all. At the beginning of 2000, 835 permanent staff operated from the Ruakura (Hamilton), Grasslands (Palmerston North), Wallaceville (Upper Hutt), Lincoln (Christchurch), and Invermay (Dunedin) campuses of Agresearch. Revenue for the year to June 2000 was $\$ 106$ million. While contract research and development remains the cornerstone of AgResearch's business, evolving into a life sciences company providing value-added high margin products and subsequent greater returns to New Zealand, is a 
major imperative. This is to be achieved by increasing revenue through the acquisition or development and delivery of new products and technologies.

This strategy necessitates the creation of new agricultural products, whose related intellectual property (IP) ownership by AgResearch must be protected by patents, trademarks (TMs), and plant variety rights (PVRs).

AgResearch (Ruakura) required a decision support system that would improve the organization's management of the costs it periodically incurs in maintaining its IP rights. We describe here the decision environment involved, and also the development and use of the DSS, called IPManager, that was constructed for this purpose. A literature review of current research into the management of intellectual property is provided in the next section. In Section 3 we discuss the use of the DSS as an OR tool. In Section 4 we discuss the DSS environment at AgResearch, and IPManager. We end the paper with some overall conclusions and a summary in Section 5.

\section{Literature on Intellectual Property Management Research}

Recently the World Intellectual Property Organization agreed to decrease patent registration fees [12]. The decreases were approved unanimously by all 171 member countries. Zarocostas [12] provides details on the amount the fees were decreased, including information on discounts for electronic applications. This news, along with the growing privatisation of research and development, has been a factor in the increasing number of patent registrations that are being applied for, on a world-wide basis. As research into the IP registration process is scarce, we provide only a brief overview of current research in this area. Beggs [2] explains the use of royalty payments, rather than fixed fees, in the licensing of patents in the presence of asymmetric information. Bousquet et al. [3] made the first formal study of risk sharing as a major rationale for the financial arrangements between a patentee and a licensee. The authors examine a particular, but empirically meaningful, class of license contracts consisting of a fixed fee, a per unit royalty, and an ad valorem royalty. The analysis proceeds by simulation in order to characterize the optimal license contracts. Lanjouw [9] has derived empirical estimates of the private value of patent protection for four technologies: computers, textiles, combustion engines, and pharmaceuticals; using new patent data for Germany. It is assumed that patentees must pay renewal fees to keep their patents in force, as well as legal expenses in order to enforce them. We now go on to discuss the DSS approach to management issues, of which IP fee management is an example.

\section{The Decision Support System as an Aid to OR Practice}

The decision support system (DSS) has emerged as a computer-based approach to assisting decision makers to address semi-structured problems by allowing them to access and use data and analytic models (Turban [11]). DSSs are interactive computerbased systems, aimed at semi-structured problems, utilising models with internal and external databases, and emphasising flexibility, effectiveness, and adaptability. These characteristics have guided much of the research in the DSS area, but the potential benefits of the DSS in the business environment are yet to be fully realized. Nevertheless, many successful DSS applications have been reported in the literature (Arinze et al. [1], Couillard [5], Parker [10]) Most of these applications are either largescale systems built to facilitate well-defined and repetitive decision tasks, or else they are 
small PC-based systems offering quick and economic routines to support one-time decision making (Islei et al. [7]). Although the definition of the DSS concept has been elusive (Bonczek et al. [4] and Er [6]), the field has flourished with the development of computer technology. Keen [8] reviewed a decade of DSS development and concluded that there is a need for a balance between each of the three DSS elements: decision, support, and systems. He felt that more research effort on the decision component was required to restore this balance, as the technology for the system component was no longer a bottleneck. To achieve "the mission of the DSS - to help people to make better decisions", Keen stressed the need for an active supporting role for "decisions that really matter". We now focus on the decision component of the DSS.

Many DSS's have the basic structure that is illustrated in Figure 1. The model and solution technique bases are included to incorporate mathematical programming (MP) techniques. Clearly, they could include all appropriate models and their companion solution techniques that may be useful in order to gain insight into the scenario for which the particular DSS is designed. These models and techniques may not necessarily be confined to the classical, deterministic models such as linear, integer, nonlinear, and dynamic programming, but also those from areas such as queuing, scheduling, inventory, and others. The models and techniques bases are included in order to be used, as necessary, to solve certain sub- issues or precise questions that arise during the overall analysis of the main scenario. They can be invoked to answer "what if?" questions, to perform sensitivity analysis, and to provide precise solutions to sub-problems that can be modelled exactly. For example within a vehicle routing DSS, a travelling salesman problem (TSP) model and various TSP solution techniques could be included. Then, if it has been established that a given vehicle will visit an identified list of clients, the TSP model and a TSP solution technique could be invoked to establish a least-distance tour.

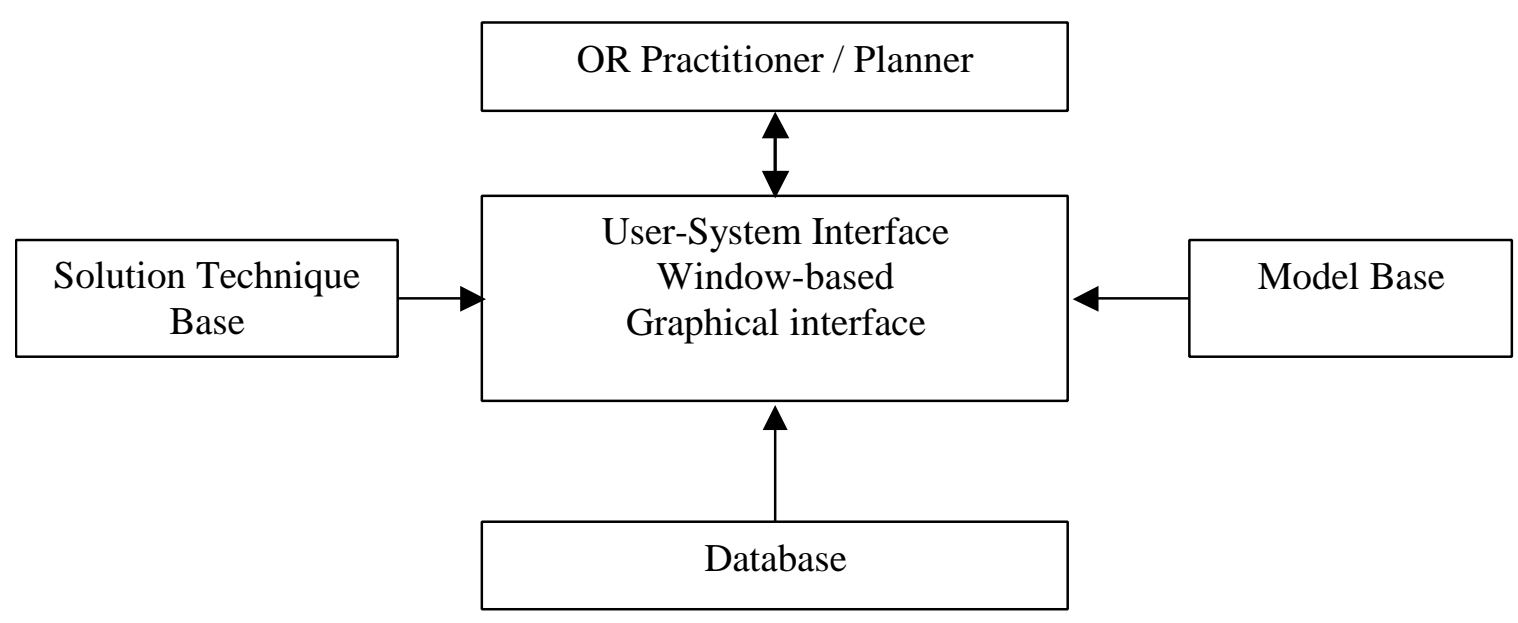

Figure 1 The Structure of a Decision Support System.

However it must be stressed that any DSS should be much more than just a mere collection of models and solution techniques. Although this can be quite a valuable aid to the implementation of MP, in terms of user friendliness and convenience, it should be only a small part of any DSS. The essence of the DSS is the user-system interface that allows the planners to: experiment, input local knowledge and inspiration, deal with unstructured situations, be flexible, allow for multiple objectives, and soft (violatable to some degree) constraints. As an example, an educational course timetabling DSS may have, as its primary purpose, the identification of a feasible timetable, without the 
optimization of any objective functions. The timetablers will "play" with the DSS, inputting various course-room-teacher-time slot combinations; noting various statistics that the DSS displays. Judgements as to the worth of various combinations are often made on grounds that difficult to quantify and virtually impossible to model. Nevertheless, suggestions can be made by the DSS, based on various assignment, matching, and allocation models and solution techniques from the bases in Figure 1.

In this section we have described the basic elements of any DSS and how appropriate OR models and solution techniques can be incorporated into it. We now go on to outline the DSS environment at AgResearch.

\section{The DSS Environment at AgResearch and IPManager}

\subsection{Overview}

We first focus on the information required to manage the costs associated with the IP protection process. IP information consists of events, associated dates, and associated costs. For example, an IP examination in a specific country is one such event. Thus one needs to know what events (with dates and costs) that have occurred so far, and what events are planned in the future in order to estimate the total costs. Even though the actual occurrences of events and costs cannot be known in advance, an estimated schedule of events and costs is available for each item of IP maintained by AgResearch. The schedule is also differentiated by the particular countries in which AgResearch maintains IP rights.

A schedule for New Zealand patent registration can be represented in the following manner. The events and timing are shown in Figure 2. In addition an annual maintenance fee is payable until the IP right is granted. Then a renewal fee is payable after $1,4,7$, and 10 years. Obviously, the costs and timings are expectations only, but they permit future costs to be estimated. IP applications can be discontinued at any time thus avoiding any costs thereafter. There is also a statutory term limit of 20 years for the patent rights in New Zealand.

Patents in foreign countries may be registered in one of two ways: 1) by applying directly to a country, based solely on the New Zealand application date, or 2) by applying to the country on the basis of the patent cooperation treaty (PCT). The second process simplifies work in foreign countries, but a prior PCT application needs to be made in New Zealand first.

\subsection{Applying Directly to a Foreign Country}

Figure 3 shows the events, costs, and expected timings of the process of applying for patents directly in Canada. In addition there are annual maintenance fees before the grant, and renewal fees after the grant. The events may be slightly different from country to country. Each country has its own schedule of maintenance and renewal fees. For example, in the USA, renewal fees are payable 4, 8, and 12 years after the patent grant. 


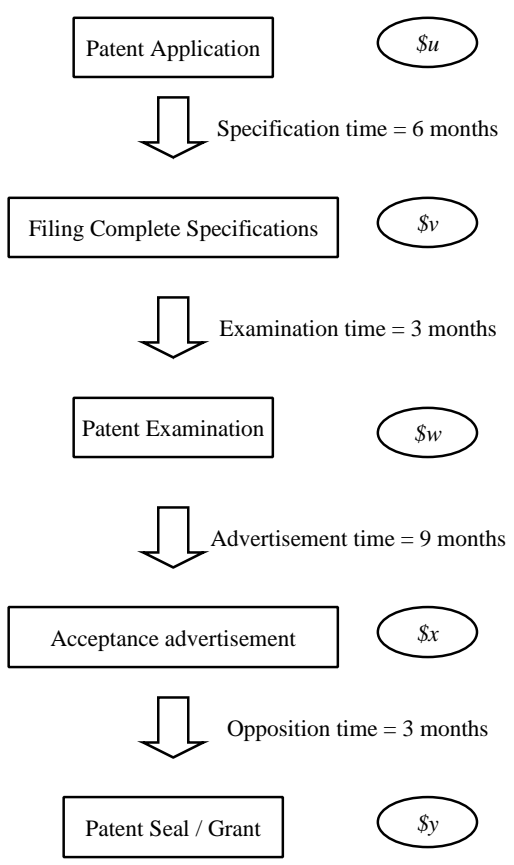

Figure 2 A New Zealand Patent Registration Schedule.

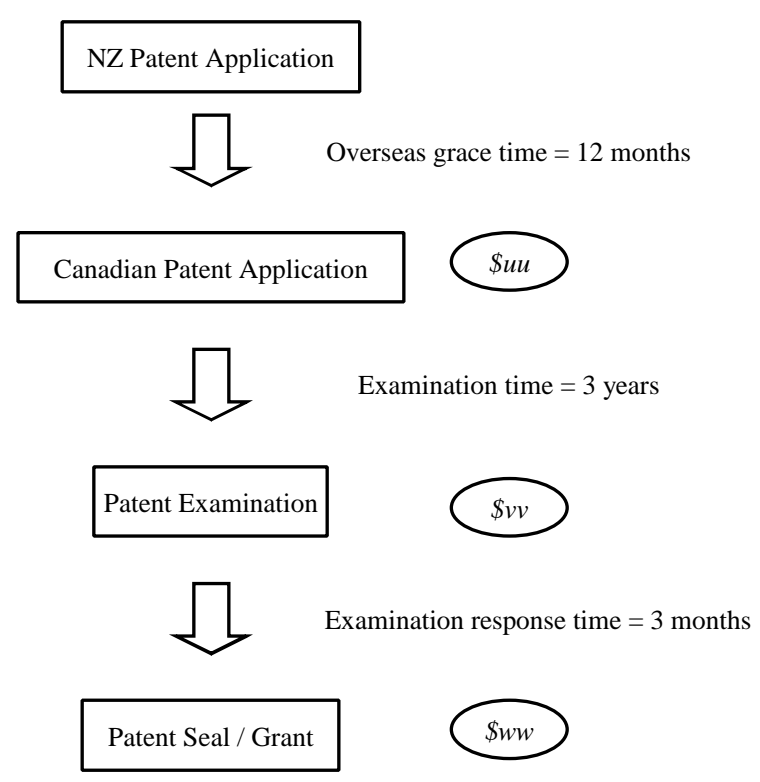

Figure 3 Direct Patent Application Process in a Foreign Country.

\subsection{Applying via the PCT}

Patent application via the PCT occurs in two stages. The PCT procedure occurs first in New Zealand. An international preliminary examination is carried out by the New Zealand Patent Office, acting as an agent for the World Intellectual Property Organisation (WIPO). Once this stage is complete, the patent application is processed in individual countries (the national phase). The PCT application, until it enters the national phase, is shown in Figure 4.

Once the PCT application has entered the national phase, application is made in individual countries. Once again, there are maintenance and renewal fees, with their own schedules of time and amount. There are also time limits for patents specific to each country.

\subsection{Trademarks and Plant Variety Rights}

So far the discussion has focussed on patents, but the application process for TMs and PVRs follow similar patterns. Both these processes essentially consist of application, examination, and registration. However, the events, their schedules, and their costs are different. There is no PCT application process for both trademarks and PVRs. All overseas applications have to be made directly to the individual countries. A single application suffices for PVR in the European Community. Similar to patents, there are maintenance and renewal fee schedules and time limits for trademarks and PVRs for each country. 


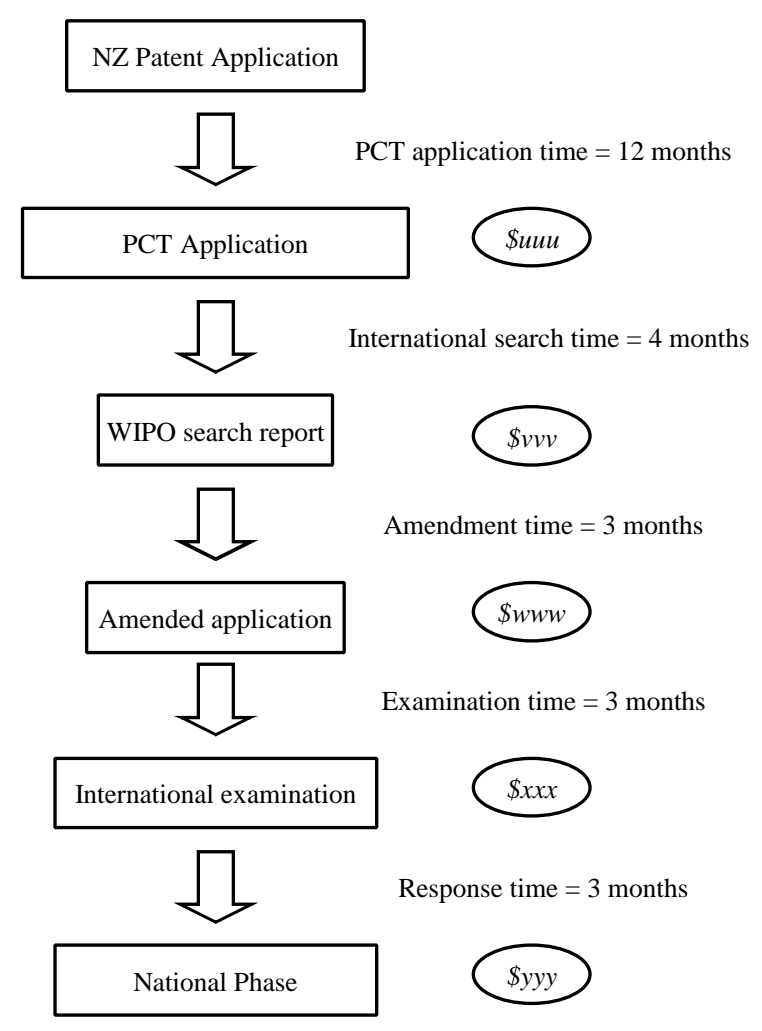

Figure 4 PCT Application Process.

\subsection{Decision Support}

To support decisions, such as when to file applications or when to abandon an IP process, a schedule of expected events for each country for each IP type is needed. Once this is available, and a series of tentative decisions are made (such as whether to apply for a PCT or whether to apply to 5 or 15 European countries), one can apply the schedules to the decisions and consequent yearly costs can be estimated. IPManager was devised to help AgResearch manage the fees that the organization pays concerning their IP rights. It provides estimates of future expenses incurred in pursuing, registering, maintaining, and renewing IP rights in various countries. These estimates incorporate prior commitments and provide decision support to AgResearch concerning desirable future courses of action to be taken with regards to IP financial strategies. In addition, IPManager maintains information on the current registration status and past costs of IP. This information consists of events, associated dates, and associated costs. For example, an IP examination in a specific country is one such event. Thus one needs to know what events (with dates and costs) have occurred so far, and what events are planned in the future, with respect to prior and new IP, in order to estimate the total costs.

Even though the actual occurrences of events and costs cannot be known in advance, an estimated schedule of events and costs is available for each of the three kinds of IP: patents, trademarks, and plant variety rights. The schedule is also differentiated by the countries where the IP rights are registered. Management can thus explore different IP management scenarios to meet budget allocations and ensure current plans do not jeopardise future opportunities.

IPManager enables the decision maker to start with a projected future schedule of events, based on the default schedules, as explained above. However this schedule must 
be updated individually, with the actual dates, as the relevant events occur. These updated records not only provide decision support as explained above, but also provide reports, such as reminders for renewal and for other pending actions.

\subsection{Maintaining Records}

Default schedules exist for each IP type (patent, TM, or PVR) for each application path (direct or via PCT), but these are only estimates. The actual realizations may well be significantly different. For example, the estimated application examination time may be 16 months in a certain country, but actually it may be completed in 24 months. This then impacts the timing of all the remaining events in that application path. Similarly, the grace period for an amended claim may be 6 months, but the decision maker may elect to submit the amended claim in 2 months, thus affecting all of the following events.

\subsection{DSS Facilities}

IPManager provides the following facilities: updating records for each IP, deciding to abandon or continue an application path, deciding to time IP actions early or later, where such choices exist, and deciding to start or continue applications in particular countries.

\subsection{Screens and Reports}

IPManager provides the following screens and reports: reminder of fees to be paid, annual projected budget (total across all IPs, for each IP, for each IP type, for each country), lists of current IP items (patents, trademarks, PVRs ), estimates of future expenses regarding IP rights (for each IP item: pursuit, application filing, examination, and acceptance advertisement, seal granting, registration (direct and PCT), maintenance, renewal, discontinuance, current registration status, recording of actual incurred past events, time spans, and associated costs that have occurred, prediction of events, time spans, and associated costs to come, updating of events, time spans, and associated costs to come resulting from changes due to the occurrence of actual events, time spans, and costs incurred), "what-if?" scenario analysis, decision support for IP activity (continuance with certain existing IP rights, allowance of certain existing IP rights to lapse, selection of countries for each new, or ongoing, IP item, with a schedule of costs, timing decisions regarding accelaration or delay of actions), yearly budget limit comparison, database of current IP and costs, IP costs by appropriate time units, cashflow, a separate window for each IP (with full details), links to the research outputs database of AgResearch, differentiation of IP types (patents, TMs, PVRs), differentiation according to country, screens and reports (reminders of future costs, annual projected budget (across all IP items, for each IP, for each IP type, for each country)), lists of current IP according to type. Figure 5 shows a screen that enables the planning of IP decisions by country (the information is disguised).

We have described the IP decision environment at AgResearch, and the DSS that was developed to aid in the management of the events and costs that relate to that environment. We now present some concluding remarks and a summary. 


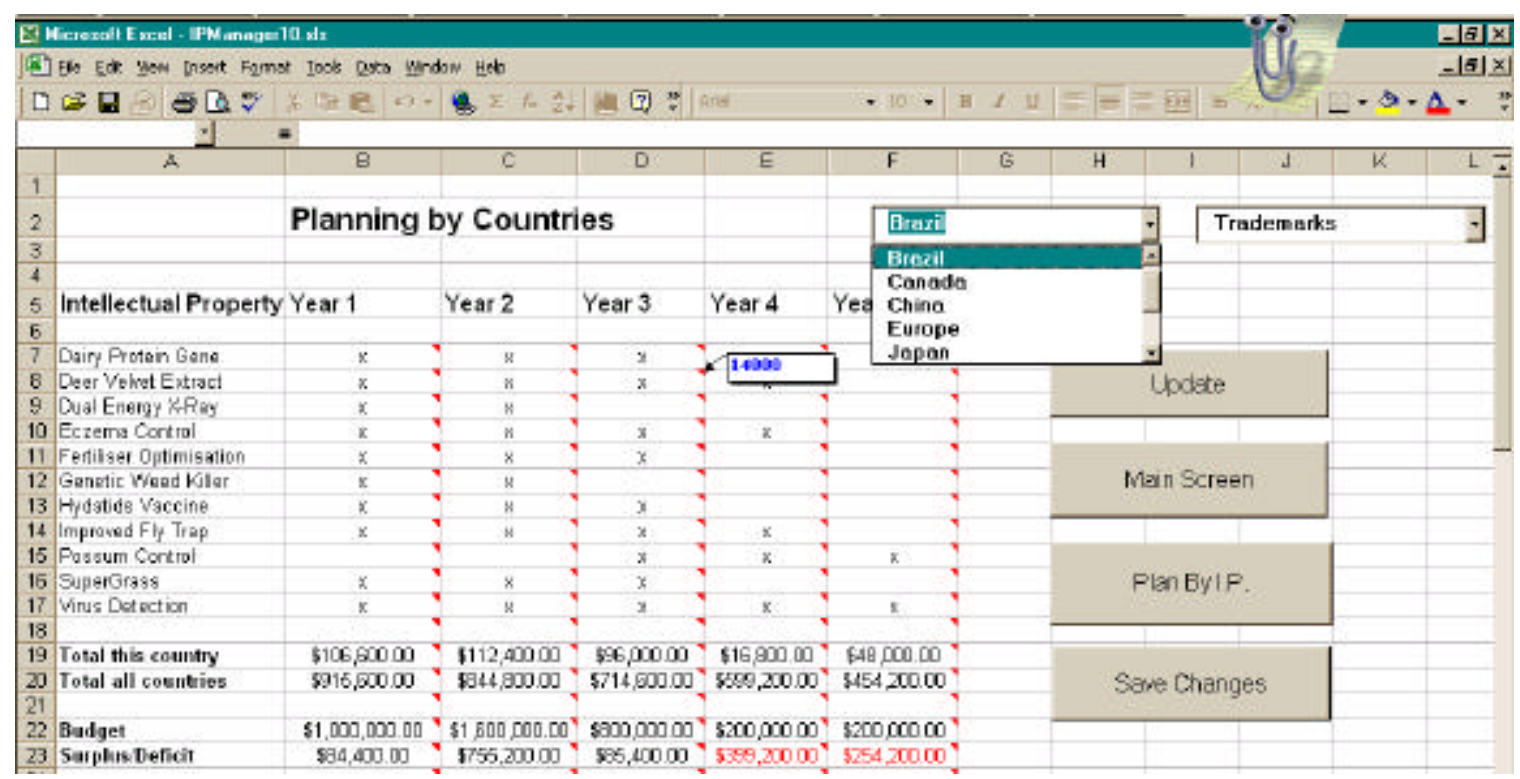

Figure 5 IP Planning Screen

\section{Conclusions and Summary}

We introduced a DSS, called IPManager, which is designed to address issues concerning the management of the events and fees that are incurred in the process of protecting intellectual property rights. IPManager seeks to assist financial planners and controllers at every step of this process. It does not automate the decision making process but helps planners by providing powerful tools to create schedules, choose between plans, generate alternative plans, and to assess alternative plans with respect to given criteria. In general, DSS benefits are often uncertain and are difficult to assess. In our case, with the prototyping approach, where development was evolutionary, this is especially the case. The ongoing schedule changes and changing environments make it even more so. The true value of a DSS is whether it improves a manager's decision making, which is not easily measured. Therefore, the traditional cost benefit analysis will not be able to capture all DSS benefits. Actually in some cases, it may not be well suited to the DSS. However, some of the benefits in our case can be measured, such as planning time. The system also has tangible and intangible benefits. It can benefit the planners in the fine tuning of existing schedules, creation of entirely new schedules, strategic planning for IP items, and the flexibility to plan for and cope with unexpected situations. The DSS also allows the planner to carry out ad hoc analysis through "what if?" queries. It also provides the scheduler with a better understanding of the relevant issues, where the system can alert users concerning illogical outcomes, such as budget blowouts. In summary, this paper shows that IP protection and management is a complex process, involving large amounts of subjective and objective information and requiring expert judgments. IPManager is designed to support research managers in coping with such complex situations. 


\section{References}

[1] B. Arinze, M. Igbaria, and L.F. Young, A Knowledge Based Decision Support System for Computer Performance Management, Decision Support Systems, 8 (1992), pp 501-515.

[2] A.W. Beggs, The Licensing of Patents Under Asymmetric Information, International Journal of Industrial Organization, 10 (1992), pp 171-191.

[3] A. Bousquet, H. Cremer, M. Ivaldi, and M. Wolkowicz, Patent Licensing and Risk Sharing - Simulation Results, Annales Des Telecommunications, 50 (1995), pp 297305 .

[4] R.H. Bonczek, C.W. Holsapple, and A.B. Whinston, Foundations of Decision Support Systems, Academic Press, New York (1981).

[5] J. Couillard, A Decision Support System for Vehicle Fleet Planning, Decision Support Systems, 9 (1993), pp 149-159.

[6] M.C. Er, Decision Support Systems: A Summary, Problems, and Future Trends, Decision Support Systems, 4 (1988), pp 355-363.

[7] G. Islei, G. Lockett, B. Cox, S. Gisbourne, and M. Stratford, Modelling Strategic Decision-Making and Performance Measurement at ICI Pharmaceuticals, Interfaces, 21 (1991), pp 4 - 22.

[8] P.G.W. Keen, Decision Support Systems: The Next Decade, Decision Support Systems, 3 (1988), pp 253-265.

[9] J.E. Lanjouw, Patent Protection in the Shadow of Infringements: Simulation Estimations of Patent Value, The Review of Economic Studies, 65 (1998), pp 671710.

[10] W.J. Parker, A.E. Dooley, and C.K. Dake, Decision Support for Farm Business Strategy: An Example for Sheep Breeding, In Proceedings of the International Congress on Modelling and Simulation, L. Oxley and F. Scrimgeour (Eds), Hamilton (1999).

[11]E. Turban, Decision Support and Expert Systems: Management Support Systems, 3rd Edition, Macmillan, New York (1993).

[12] J. Zarocostas, Patent Fees Are Cut Again, National Law Journal, 21 (1998), pA13. 